\title{
Quantitative Analysis and Comparison of Flavonoids in Lotus Plumules of Four Representative Lotus Cultivars
}

\author{
Ting Liu, ${ }^{1,2}$ Mingzhi Zhu, ${ }^{1}$ Chunyun Zhang, ${ }^{1,3}$ and Mingquan Guo ${ }^{1,3}$ \\ ${ }^{1}$ Key Laboratory of Plant Germplasm Enhancement and Specialty Agriculture, Wuhan Botanical Garden, Chinese Academy of \\ Sciences, Wuhan 430074, China \\ ${ }^{2}$ University of Chinese Academy of Science, Beijing 100049, China \\ ${ }^{3}$ Sino-African Joint Research Center, Chinese Academy of Sciences, Wuhan 430074, China \\ Correspondence should be addressed to Mingquan Guo; guomq@wbgcas.cn
}

Received 9 May 2017; Revised 27 July 2017; Accepted 23 August 2017; Published 26 September 2017

Academic Editor: Maria del Mar Contreras

Copyright ( 2017 Ting Liu et al. This is an open access article distributed under the Creative Commons Attribution License, which permits unrestricted use, distribution, and reproduction in any medium, provided the original work is properly cited.

\begin{abstract}
The flavonoids in lotus plumules from four representative lotus cultivars have been analyzed using high-performance liquid chromatography coupled with ultraviolet detector and electrospray ionization triple quadrupole mass spectrometry. By this means, sixteen flavonoids were successfully measured and compared among four cultivars. Although similar flavonoid compositions were detected from these four cultivars, their flavonoid contents were significantly different. cv. Bailian from Guangchang was detected to have the most total flavonoid content, that is, $1595.86 \mathrm{mg} / 100 \mathrm{~g}$, followed by cv. Xuanlian from Wuyi $(1553.49 \mathrm{mg} / 100 \mathrm{~g})$, cv. Xianglian from Xiangtan $(1173.07 \mathrm{mg} / 100 \mathrm{~g})$, and cv. Jianlian from Jianning $(930.08 \mathrm{mg} / 100 \mathrm{~g})$. However, similar percentages of flavonoid $C$-glycosides in the total flavonoids were found for four cultivars, which were $80.83 \%$ for cv. Bailian, $80.91 \%$ for cv. Xianglian, 79.25\% for cv. Jianlian, and 78.53\% for cv. Xuanlian. This work will be very useful for quality assurance and control for the lotus plumules from different origins in terms of qualitative and quantitative information about flavonoids. It will also be of special interest in the screening of lotus plumules with high flavonoid content, which are preferred due to their wide potential applications in food and pharmaceutical industries.
\end{abstract}

\section{Introduction}

Nenumbo nucifera is a valuable aquatic economic plant, and widely distributed in China [1]. It has more than 600 cultivars [2] and the four representative ones distributed in Hunan (cv. Xianglian), Jiangxi (cv. Bailian), Zhejiang (cv. Xuanlian) and Fujian (cv. Jianlian) province are the four most famous cultivars in Chinese history. All tissues of Nenumbo nucifera can be used as common foods or traditional medicines; so it is called the "full body of treasures" $[3,4]$. Lotus plumule, also called Lianzixin, is the green seed embryo of the lotus seed. The mature lotus plumule has a bitter taste, and been recorded by Chinese Pharmacopoeia (2010 version) as a traditional Chinese medicine. Lotus plumule is also approved for dual purposes as a medical and edible plant by the Chinese Ministry of Health [5]. It is of important medical value, and could be used for the treatment of heart heat, high blood pressure and high fever [6]. It is also used as a healthcare food. In southern China, lotus plumules have been drunk as tea with boiling water. Xiao [7] developed a kind of health beverage made up of lotus plumules and Chrysanthemum in recent years. Lotus plumules, together with green tea and other materials, are also developed into tea beverage. With more and more lotus plumules derived products into the market, there is growing demand for raw materials of lotus plumules. Thus, quality assurance and control for raw materials and the derived products of lotus plumules are in great need, especially their natural phytochemicals and bioactive components, which may vary in cultivars from different districts. As it is known, the phytochemicals and bioactivities of plants are closely related to both the genetic strains and the environment factors including the insolation, temperature, precipitation, and other factors $[8,9]$. For example, flavonols biosynthesis 
is stimulated by sunlight exposure, and an increased content of flavonols means high antioxidant potency, and thus more bioactivities $[10,11]$.

It has been reported that alkaloids and flavonoids are the two major types of bioactive compounds in lotus plumule together with polysaccharide. Alkaloids in lotus plumule are main liensiline, neferine and isoliensiline [12]. Early studies revealed that the biological activities of these alkaloids include antihypertensive, hypoglycemic and anti-human immunodeficiency effects, and so on $[13,14]$. Comparing to alkaloids, very limited studies focusing on the composition and pharmacological properties of flavonoids in lotus plumule have been reported. In recent years, Li et al. [15] identified eight flavonoid $\mathrm{C}$-glycosides and one flavonoid $\mathrm{O}$ glycoside in lotus plumule for the first time. Compared with alkaloids, Chen et al. [16] and Li et al. [15] found that lotus plumule has a much higher content of total flavonoid. In recent years, Zhu et al. [17] and Feng et al. [14] reported that flavonoids in lotus plumule exhibited good potential of antioxidant activity. It is thus more favorable and practical to select flavonoids in lotus plumule as the main phytochemical components for the quality assurance and control purpose. To the best of our knowledge, the total content and individual content of flavonoids of lotus plumule obtained from different cultivars and different districts have yet been evaluated. In this study, we evaluated lotus plumules of four representative cultivars (cv. Bailian, Xuanlian, Xianglian, Jianlian), which were collected from four different districts. Their total and individual content of flavonoids were determined by combining macroporous resin chromatography with high-performance liquid chromatography (MPRCHPLC) method, which was developed for the simultaneous enrichment and analysis of flavonoids from lotus leaves, recently [18]. It would be of key importance for the following reasons. On the one hand, it could be very useful for quality assurance and control purpose for the lotus plumules from different origins. On the other hand, it can also be used to screen for the best cultivar of lotus plumules with high flavonoid content for better and wider cultivation and applications.

\section{Materials and Methods}

2.1. Materials, Chemicals, and Reagents. Jiangxi, Hunan, Fujian, and Zhejiang are four representative places for the production of lotus plumules. Lotus plumules from Guangchang $\left(\mathrm{GC}, 116^{\circ} 35^{\prime} \mathrm{E}, 26^{\circ} 84^{\prime} \mathrm{N}\right)$ in Jiangxi province, called cv. Bailian, were purchased from a commercial source. The other three samples, called cv. Xianglian, cv. Jianlian, and cv. Xuanlian, were collected from Xiangtan (XT, $112^{\circ} 93^{\prime} \mathrm{E}$, $27^{\circ} 90^{\prime} \mathrm{N}$ ) in Hunan province, Jianning (JN, $116^{\circ} 84^{\prime} \mathrm{E}, 26^{\circ} 83^{\prime}$ $\mathrm{N})$ in Fujian province, and Wuyi (WY, $119^{\circ} 81^{\prime} \mathrm{E}, 28^{\circ} 90^{\prime} \mathrm{N}$ ) in Zhejiang province, respectively. Those samples were collected from their native districts and authorized by the taxonomist Professor Guangwan $\mathrm{Hu}$ from the Key Laboratory of Plant Germplasm Enhancement and Specialty Agriculture, Chinese Academy of Sciences. Raw materials were powdered and stored under dry, dark, and closed condition until use. Standards of apigenin-6-C-glucosyl-8-C- arabinoside (schaftoside), apigenin-6- $C$-arabinosyl-8- $C$-glucoside (isoschaftoside), and quercetin-3-O-glucoside (isoquercitrin) were obtained from Tongtian Tauto Biotech Company (Shanghai, China). Rutin was purchased from J\&K Scientific Ltd. (Beijing, China). The four standards above were all of $95 \%$ purity. They were stored in the fridge at $4^{\circ} \mathrm{C}$. Acetonitrile for HPLC and MS was of LC-grade and from Fisher (Pittsburgh, PA, USA); formic acid (99\%) was of LC-grade and from ROE (ROE scientific, USA); and deionized water was prepared using an EPED water purification system (Nanjing, China).

2.2. Instrument and Chromatographic Conditions. The quantification of flavonoids was carried out with the method of HPLC-UV on a Thermo Accela 1250 HPLC system (Thermo Fisher Scientific, San Jose, CA, USA) including an autosampler, a 1250 pump, a vacuum degasser, and an UV/Vis detector. An aliquot of $10 \mu \mathrm{L}$ was injected to the HPLC system and analyzed. Chromatographic separation was performed on a Welch Ultimate XB-C18 column $(150 \mathrm{~mm} \times 2.1 \mathrm{~mm}, 5 \mu \mathrm{m})$. A mobile phase system consisted of water with $0.5 \%$ formic acid (A) and acetonitrile with $0.1 \%$ formic acid (B). Gradient elution, at a flow rate of $0.3 \mathrm{~mL} / \mathrm{min}$, was set as follows: $0-10 \mathrm{~min}$ at $12 \% \mathrm{~B} ; 10-42 \mathrm{~min}$, from $12 \%$ to $20 \% \mathrm{~B}$; $42-52 \mathrm{~min}$, from $20 \%$ to $30 \% \mathrm{~B}$; and $52.1-55 \mathrm{~min}$ at $12 \% \mathrm{~B}$. The wavelength of $350 \mathrm{~nm}$ was chosen and the column temperature was set at $30^{\circ} \mathrm{C}$ after optimization. The flavonoid contents were calculated on peak areas obtained from UV and calibrated by standards.

For the identification of flavonoids, HPLC-MS/MS analyses were carried out by a thermo 600 HPLC system (Thermo Fisher Scientific, San Jose, CA, USA) comprising of an autosampler, a 600 pump, and an UV/Vis detector coupled to an electrospray ionization triple quadrupole mass analyzer (Thermo Fisher Scientific, San Jose, CA, USA) and conducted in the negative ionization (NI) mode. The LC conditions were the same as mentioned above. Some other major parameters were as follows: spray voltage, $3000 \mathrm{~V}$; vaporizer temperature, $300^{\circ} \mathrm{C}$; sheath gas $\left(\mathrm{N}_{2}\right)$ pressure, 40 arbitrary units; aux gas pressure, 10 arbitrary units; capillary temperature, $350^{\circ} \mathrm{C}$; collision energy, $15 \mathrm{~V}$; and scan range, $m / z 150-1000$.

2.3. Preparation of Standard Solutions. Four standards were accurately weighed and dissolved in methanol to achieve a concentration of $1 \mathrm{mg} / \mathrm{mL}$, respectively. Working solutions were prepared by combining four stock solutions and diluting with methanol/water $(50: 50, v: v)$ to seven different concentrations. Before HPLC analysis, each working solution was centrifuged at $8000 \mathrm{rpm}$ for $5 \mathrm{~min}$ with a centrifuge 5180R (Eppendorf, Germany).

2.4. Preparation of Sample Solutions. As referred to Zhu et al. [18] with some minor modifications, $20 \mathrm{~g}$ of dried powders of lotus plumule was ultrasonically extracted with $200 \mathrm{~mL}$ of $70 \%$ ethanol for $40 \mathrm{~min}$ and then the extracts were filtered with filter paper. The residues were re-extracted twice and filtered. The combined solution was evaporated to dryness under reduced pressure at $50^{\circ} \mathrm{C}$ using a rotary evaporator 


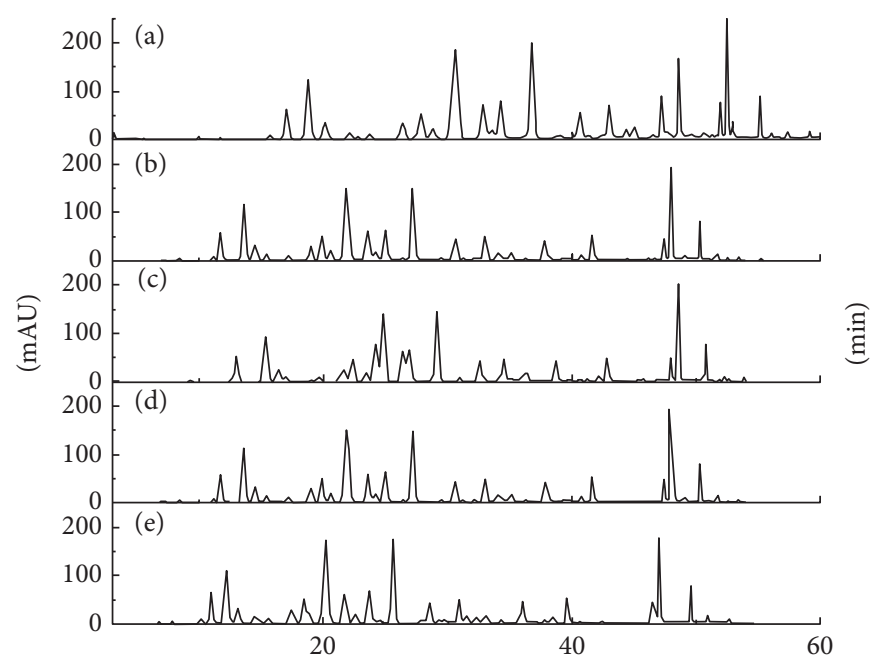

Figure 1: Optimization of LC flow rate and column temperature using the sample eluted with $50 \%$ ethanol from extracts of lotus plumule from XT: (a) $0.2 \mathrm{~mL} / \mathrm{min}$; (b) $0.3 \mathrm{~mL} / \mathrm{min}$; (c) $25^{\circ} \mathrm{C}$; (d) $30^{\circ} \mathrm{C}$; (e) $35^{\circ} \mathrm{C}$.

TABLE 1: Regression equation, regression coefficients, linearity, LOD, and LOQ of four flavonoid standards.

\begin{tabular}{llcccc}
\hline Compounds & Regression equation & $R^{2}$ & Linear range $(\mu \mathrm{g} / \mathrm{mL})$ & LOD $(\mu \mathrm{g} / \mathrm{mL})$ & 0.18 \\
Isoschaftoside & $y=63731 x+32602$ & 1.00 & $2-250$ & 0.12 & 0.61 \\
Schaftoside & $y=64167 x+19892$ & 1.00 & $2-125$ & $2-250$ & 0.17 \\
Rutin & $y=37430 x+8145$ & 1.00 & $2-125$ & 0.17 & 0.40 \\
Isoquercitrin & $y=46049 x+14264$ & 1.00 & 0.59 \\
\hline
\end{tabular}

RE-52AA (Shanghai, China). Then, part of the resultant residues were loaded to a macroporous resin column with adsorption resin D101 (Nan Kai University, Tianjin) to further enrich and purify the flavonoids of interest. The loaded column was eluted successively with $1000 \mathrm{~mL}$ of water and 30\%, 50\%, 70\%, and 90\% ethanol. Each fraction was evaporated to almost dryness except for the water part. All parts were dissolved with $50 \mathrm{~mL}$ of methanol and then centrifuged at $8000 \mathrm{rpm}$ for $8 \mathrm{~min}$. The supernatants were transferred to a $50 \mathrm{~mL}$ volumetric flask and diluted with methanol/water $(50: 50, v: v)$ to a suitable concentration before HPLC analysis.

\section{Results and Discussion}

3.1. Optimization of LC Conditions. The chromatographic separation condition was referred to Zhu et al. [18] and Chen et al. [16] and some modifications were made to achieve a faster and better separation. Lotus plumules from XT (Xiangtan in Hunan province) were used to optimize chromatographic conditions. It was found that the concentration of formic acid as an additive had an important impact on the separation of flavonoids, and the same concentration of formic acid was chosen according to the optimized condition by Chen et al. [16]. Subsequently, the flow rate $(0.2 \mathrm{~mL} / \mathrm{min}, 0.3 \mathrm{~mL} / \mathrm{min})$ and column temperature $\left(25^{\circ} \mathrm{C}\right.$, $30^{\circ} \mathrm{C}$, and $\left.35^{\circ} \mathrm{C}\right)$ were investigated. In case of the effects of flow rate, there were no differences on the separation of flavonoids, but the required analysis time was around five minutes shorter at the flow rate of $0.3 \mathrm{~mL} / \mathrm{min}$ than of $0.2 \mathrm{~mL} / \mathrm{min}$ as shown in Figure $1(\mathrm{a}$ and $\mathrm{b})$. In terms of column temperature, by comparing the LC chromatograms of different temperatures at the flow rate of $0.3 \mathrm{~mL} / \mathrm{min}$, $30^{\circ} \mathrm{C}$ was found to achieve a better resolution and peak shape than those of the other two conditions as exhibited in Figure 1(c to e). As a result, water containing $0.5 \%$ formic acid, acetonitrile containing $0.1 \%$ formic acid, flow rate of $0.3 \mathrm{~mL} / \mathrm{min}$, and a temperature of $30^{\circ} \mathrm{C}$ were employed in the following studies.

3.2. Method Validation. Working solutions were tested in triplicate under the concentration gradients. The calibration curves of four standards all showed good linearity at the concentration range $\left(r^{2}=1.00\right)$. The limit of detection (LOD) and the limit of quantification (LOQ) were calculated based on the signal-to-noise ratios as 3 and 10, respectively $[19,20]$. The regression equation, LOD and LOQ of four standards (i.e., isoschaftoside, schaftoside, rutin, and isoquercitrin) were shown in Table 1.

To determine the precision of the method, three independent sample solutions (sample from XT) were analyzed. The relative standard deviation (RSD) values of peak area of thirteen peaks were from $0.58 \%$ to $4.1 \%$, indicating that the method was precise. The stability of the sample solution was determined by analyzing the same sample solution (sample from XT) at $0,4,8,12$, and $24 \mathrm{~h}$. As a result, the 
RSD values of peak area were at the range from $0.42 \%$ to $6.6 \%$, indicating that the sample solution was stable. The recovery tests were used to evaluate the accuracy of quantification, which were conducted by spiking the test sample with three different concentrations of rutin, that is, $80 \%, 100 \%$, and $120 \%$, compared to the original concentration of rutin in the test sample. Three lotus plumule samples with known amounts of flavonoids were extracted and purified as mentioned above. Each concentration was tested in triplicate. The mean values of recovery rate were $82.11 \%$ for low concentration, $83.91 \%$ for medium concentration, and $83.05 \%$ for high concentration, which were acceptable values.

3.3. Identification of Flavonoids in Lotus Plumule. Flavonoid $\mathrm{O}$-glycosides and $\mathrm{C}$-glycosides can be distinguished by means of LC-MS/MS. For flavonoid O-glycosides, heterolytic cleavage of hemiacetal $\mathrm{O}-\mathrm{C}$ bond often occurs with loss of the sugar moiety. As to flavonoid $C$-glycosides, cross-ring cleavages of the sugar moiety are common to produce characteristic ions $[\mathrm{M}-\mathrm{H}-150]^{-}\left({ }^{0,1} \mathrm{X}\right),[\mathrm{M}-\mathrm{H}-120]^{-}\left({ }^{0,2} \mathrm{X}\right)$, and $[\mathrm{M}-\mathrm{H}-90]^{-}\left({ }^{0,3} \mathrm{X}\right)$ for hexose substitution [21]. Flavonoids were identified in this study by comparing the retention time and MS/MS data with those of corresponding standards or reported references. MS/MS data in the NI mode are listed in Table 2.

3.3.1. Identification of Flavonoid O-Glycosides. Identification of flavonoid $\mathrm{O}$-glycosides by MS/MS is easy due to the direct loss of sugar moiety from the aglycone in the spectrum. In this way, seven flavonoid $O$-glycosides are detected and identified in this study. The full separation is difficult to achieve because of the similar polarity of some flavonoid compounds. Li et al. [15] used a mobile phase system of water containing 10\% formic acid and formic acid-acetonitrilewater $(10: 40: 50, v: v: v)$ to separate flavonoid compositions but failed to achieve simultaneous separation of two peaks. Similar to the previous study, peak 3 and peak 7 in this study contained two and three compounds after identification by LC-MS/MS, respectively. Peak 7 showed two $[\mathrm{M}-\mathrm{H}]^{-}$ions at $\mathrm{m} / z 609$ and $\mathrm{m} / z 463$ in the NI mode. The former produced fragment ions at $\mathrm{m} / \mathrm{z} 300\left[\mathrm{Y}_{0}-1\right]^{-}$and $\mathrm{m} / \mathrm{z} 301$ $\left[\mathrm{Y}_{0}\right]^{-}$by a loss of $308 \mathrm{Da}$ implying that it could be quercetin derivatives and thus tentatively identified as quercetin-3-Orutinoside (rutin) as reported in a previous article [22]. Similarly, $[\mathrm{M}-\mathrm{H}]^{-}$ions at $\mathrm{m} / \mathrm{z} 463$ also produced fragment ions at $m / z 300\left[\mathrm{Y}_{0}-1\right]^{-}$and $m / z 301\left[\mathrm{Y}_{0}\right]^{-}$indicating a hexose substitution of quercetin and was identified as quercetin3-O-galactoside (hyperoside). Peak 8 showed the same $[\mathrm{M}-\mathrm{H}]^{-}$ions at $\mathrm{m} / \mathrm{z} 463$ and fragment ions at $\mathrm{m} / \mathrm{z} 300$ $\left[\mathrm{Y}_{0}-1\right]^{-}$and $\mathrm{m} / \mathrm{z} 301\left[\mathrm{Y}_{0}\right]^{-}$with those of hyperoside and could be identified as quercetin-3-O-glucoside (isoquercitrin) according to their retention times. The above identification of hyperoside and isoquercitrin was also confirmed by comparing their LC-MS spectra with those of authentic standards (Figure 2c).

Peaks 10 and 11 exhibited the same $[\mathrm{M}-\mathrm{H}]^{-}$ions at $\mathrm{m} / \mathrm{z}$ 593 and fragment ion at $m / z 285\left[\mathrm{Y}_{0}\right]^{-}$, indicating that they are isomers. The ions at $m / z 285\left[\mathrm{Y}_{0}\right]^{-}$, resulting from a neutral loss of $308 \mathrm{Da}$ from the $[\mathrm{M}-\mathrm{H}]^{-}$ions, indicated that they could be derivatives of luteolin or kaempferol with a disaccharide substitution. A fragment ion at $\mathrm{m} / \mathrm{z} 447$ was more obvious in peak $10(8 \%)$ than in peak 11 , which implied that the disaccharide was deduced to be neohesperidose in peak 10 and rutinose in peak 11, respectively. Peak 10 was successfully identified to be luteolin-7-O-neohesperidoside by comparing its LC-MS with the pure compound isolated from lotus plumule (Figure 2b), while peak 11 is tentatively identified as luteolin-7-O-rutinoside according to a previous report [15]. Peak 12 showed a $[\mathrm{M}-\mathrm{H}]^{-}$ion at $\mathrm{m} / \mathrm{z} 623$ and produced ions at $\mathrm{m} / \mathrm{z} 315\left[\mathrm{Y}_{0}\right]^{-}$and $\mathrm{m} / \mathrm{z} 314$ $\left[\mathrm{Y}_{0}-1\right]^{-}$, resulting from a loss of $308 \mathrm{Da}$, which suggested that it could be $O$-diglycoside of isorhamnetin. Peak 12 was also further identified as isorhamnetin-3-O-rutinoside by comparing its LC-MS with the pure compound isolated from lotus plumule (Figure 2a). Peak 13 showed $[\mathrm{M}-\mathrm{H}]^{-}$ ions at $\mathrm{m} / \mathrm{z} 607$, which shared the same fragmentation pathways with those of peak 12, resulting in a product ion at $\mathrm{m} / z 299\left[\mathrm{Y}_{0}\right]^{-}$. It was thus identified as diosmetin7-O-rutinoside (diosmin).

3.3.2. Identification of Flavonoid C-Glycosides. Identification of flavonoid $\mathrm{C}$-glycosides is more complex than that of flavonoid $O$-glycosides due to the different MS fragmentation pathways. Herein, 9 flavonoid $C$-glycosides were detected and identified. Moreover, the fragmentation pathway of 3 detected compounds were similar to flavonoid $C$-glycosides and they were might be flavonoid $C$-glycosides which had never be found in lotus plumule. Peak 1 showed a $[\mathrm{M}-\mathrm{H}]^{-}$ ion at $m / z 593[\mathrm{M}-\mathrm{H}]^{-}$and three fragment ions at $m / z 473$ $[\mathrm{M}-\mathrm{H}-120]^{-}, \mathrm{m} / z 383[\mathrm{~A}+113]^{-}, \mathrm{m} / z 353[\mathrm{~A}+83]^{-}$. These fragment ions suggested that the aglycone is apigenin (270) substituted with two hexose $(162+162)$. It was thus identified to be apigenin 6,8-di- $C$-glucoside (vicenin-2). Peak 2 showed a $[\mathrm{M}-\mathrm{H}]^{-}$ion at $\mathrm{m} / z 563$ and four fragment ions at $\mathrm{m} / z 473[\mathrm{M}-\mathrm{H}-90]^{-}, \mathrm{m} / z 443[\mathrm{M}-\mathrm{H}-120]^{-}, \mathrm{m} / z 383$ $[\mathrm{A}+113]^{-}$, and $m / z 353[\mathrm{~A}+83]^{-}$, which implied that the aglycone is apigenin (270) substituted with a hexose (162) and a pentose (132). The relative abundance of $[\mathrm{M}-\mathrm{H}-120]^{-}$, was $5 \%$ higher than that of $[\mathrm{M}-\mathrm{H}-90]^{-}$. Peak 2 was identified as apigenin-6-C-glucosyl-8-C-xyloside $[9,18]$. As discussed above, peak 3 also gave two $[\mathrm{M}-\mathrm{H}]^{-}$ ions at $\mathrm{m} / z 563$ and $\mathrm{m} / z$ 447. Interestingly, peak 4 and peak 5 showed $[\mathrm{M}-\mathrm{H}]^{-}$ion at $\mathrm{m} / z 447$ and $\mathrm{m} / z 563$, respectively. Thus, there were two pairs of isomers existing in these three peaks. $[\mathrm{M}-\mathrm{H}]^{-}$ion at $m / z 563$ produced four fragment ions at $m / z 473[\mathrm{M}-\mathrm{H}-90]^{-}, \mathrm{m} / z 443[\mathrm{M}-\mathrm{H}-120]^{-}, \mathrm{m} / z 383$ $[\mathrm{A}+113]^{-}$, and $m / z 353[\mathrm{~A}+83]^{-}$, which was the same with those of peak 2 and could thus be identified as apigenin-6$C$-glucosyl-8- $C$-arabinoside (schaftoside) in peak 3 and apigenin-6-C-arabinosyl-8-C-glucoside (isoschaftoside) in peak 5 and be verified by comparing their LC-MS with those of authentic standards (Figure 2d). Deprotonated molecule ion at $\mathrm{m} / z 447$ produced three fragment ions at $\mathrm{m} / \mathrm{z} 357$ $[\mathrm{M}-\mathrm{H}-90]^{-}, \mathrm{m} / \mathrm{z} 327[\mathrm{M}-\mathrm{H}-120]^{-}$, and $\mathrm{m} / z 297[\mathrm{M}-\mathrm{H}$ $-150]^{-}$and could be tentatively deduced as hexose substitution of luteolin or kaempferol. In addition, the relative intensity of the ion at $m / z 357[\mathrm{M}-\mathrm{H}-90]^{-}$in peak 3 is higher than that in peak 4 [22]. $[\mathrm{M}-\mathrm{H}]^{-}$ion at $m / z 447$ in peak 3 and 


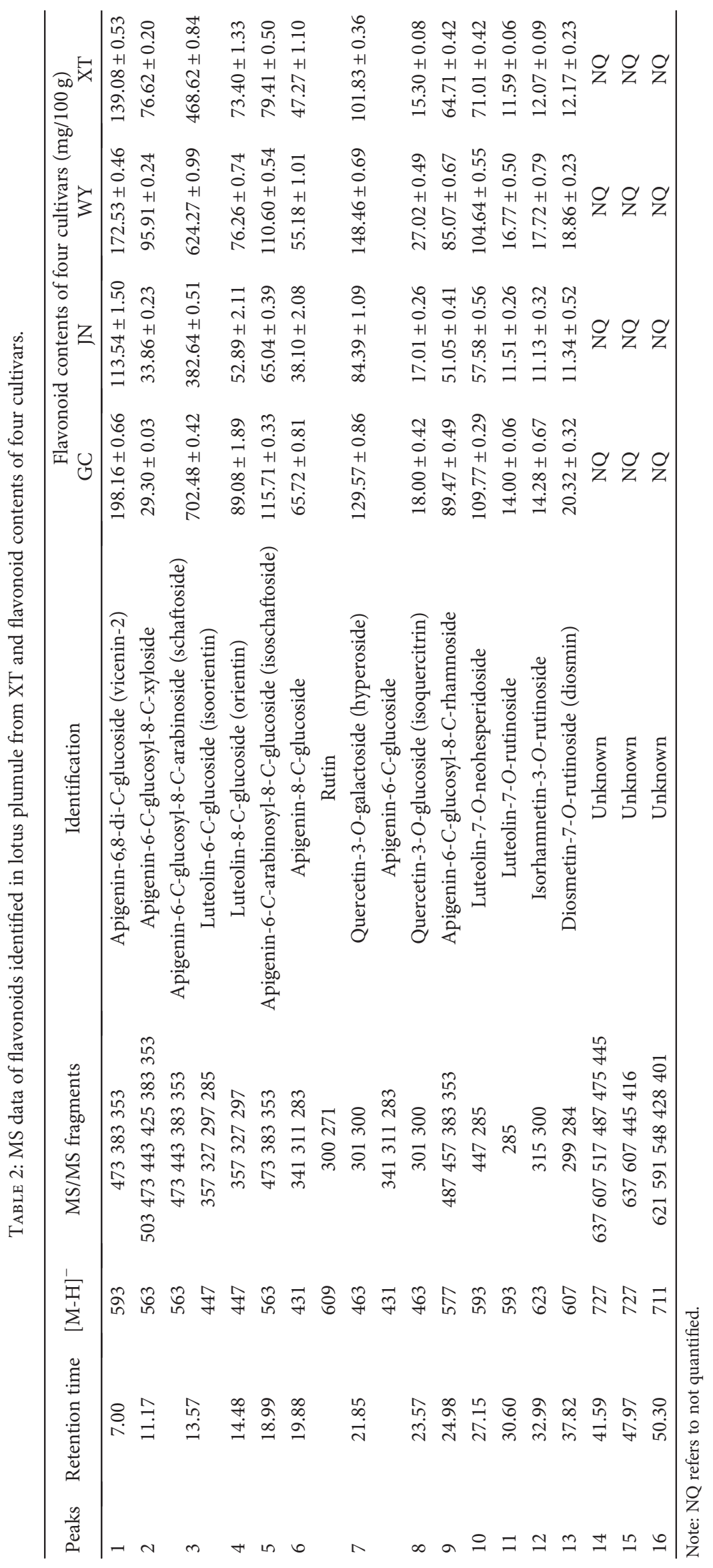




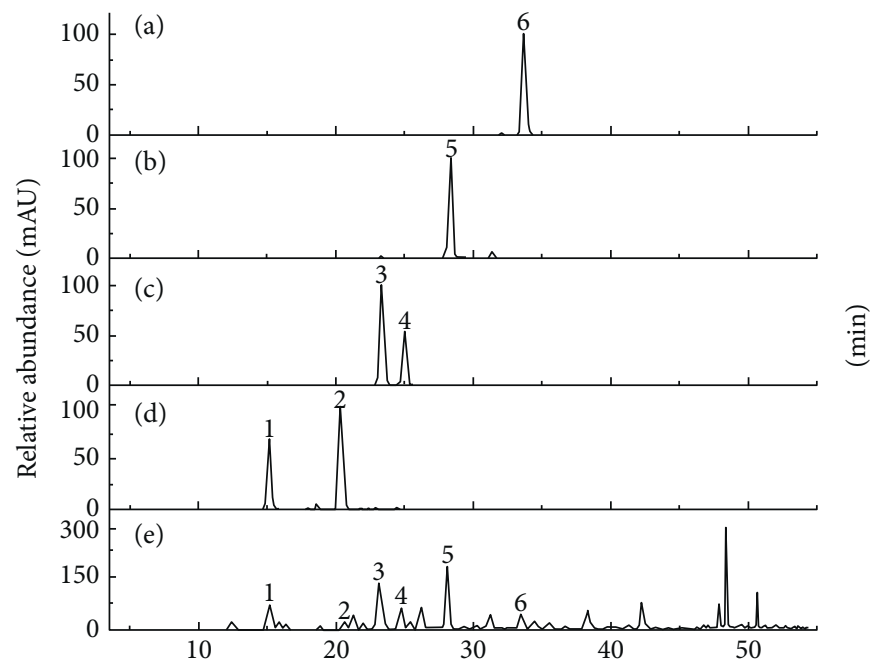

FIgURE 2: Chromatograms of LC and LC-MS for the identification of flavonoids in lotus plumule from XT: (a) isorhamnetin-3-O-rutinoside (6); (b) luteolin-7-O-neohesperidoside (5); (c) hyperoside (3) and isoquercitrin (4); (d) schaftoside (1) and isoschaftoside (2); (e) 50\% ethanol eluents.

peak 4 could thus be identified as luteolin-6-C-glucoside (isoorientin), and luteolin-8-C-glucoside (orientin), respectively. Peak 6 showed a $[\mathrm{M}-\mathrm{H}]^{-}$ion at $\mathrm{m} / \mathrm{z} 431$ and this deprotonated molecule ion also appeared in peak 7. Their fragment ions at $m / z 341[\mathrm{M}-\mathrm{H}-90]^{-}$and $m / z 311[\mathrm{M}-\mathrm{H}$ $-120]^{-}$were $16 \mathrm{Da}$ less than those of $[\mathrm{M}-\mathrm{H}]^{-}$ion at $\mathrm{m} / \mathrm{z}$ 447 , suggesting that the aglycone could be apigenin with a substitution of glucoside. Thus, they could be identified as apigenin-8-C-glucoside (vitexin) or apigenin-6-C-glucoside (isovitexin). Considering that the relative intensity of ion at $m / z 341[\mathrm{M}-\mathrm{H}-90]^{-}$was higher in isovitexin $[22,23]$, $[\mathrm{M}-\mathrm{H}]^{-}$ion at $m / z 431$ in peak 6 and peak 7 were identified as vitexin and isovitexin, respectively. Peak 9 gave a $[\mathrm{M}-\mathrm{H}]^{-}$ion at $\mathrm{m} / \mathrm{z} 577$ and produced fragment ions at $\mathrm{m} / \mathrm{z}$ $487[\mathrm{M}-\mathrm{H}-90]^{-}, \mathrm{m} / z 457[\mathrm{M}-\mathrm{H}-120]^{-}, \mathrm{m} / z 383[\mathrm{~A}+113]^{-}$ or $[\mathrm{M}-\mathrm{H}-90-104]^{-}$, and $\mathrm{m} / z 353[\mathrm{~A}+83]^{-}$or $[\mathrm{M}-\mathrm{H}-90$ $-134]^{-}$. The fragment ion at $\mathrm{m} / \mathrm{z} 457[\mathrm{M}-\mathrm{H}-120]^{-}$had a higher abundance than the fragment ion at $487[\mathrm{M}-\mathrm{H}$ $-90]^{-}$. Peak 9 could be identified as apigenin-6-C-glucosyl8-C-rhamnoside $[9,22]$.

Peaks 14-16, with the deprotonated molecule ions at $\mathrm{m} / \mathrm{z}$ $727, m / z 727$, and $m / z 711$, respectively, have not been found in any previous research on lotus, and their fragment ions are so complex that they are difficult to be identified by now. However, their characteristic fragment ions at $[\mathrm{M}-\mathrm{H}-90]^{-}$ and $[\mathrm{M}-\mathrm{H}-120]^{-}$in Table 2 suggested that they could be flavonoid $C$-glycosides.

\subsection{Total Flavonoid Content (TFC) and Individual Flavonoid} Content. Almost all of the flavonoids were enriched in $30 \%$ and $50 \%$ elution parts, and both the $30 \%$ and $50 \%$ ethanol elution were used to wash the macroporous resin column in order to achieve a better separation in the HPLC and thus a better analysis of flavonoids in lotus plumule. The chromatograms of lotus plumule from four different districts are shown in Figures 3 and 4 . The content of flavonoid was expressed as $\mathrm{mg} / 100 \mathrm{~g}$ dry weight. Compounds $3,5,7$, and
8 were quantified by using their corresponding standards, and the contents of the remaining compounds were determined using rutin as an external standard. The structure of peaks 14-16 was unknown for the time being, so the total flavonoid content just included the flavonoids of peaks 1-13. The results showed that lotus plumule from GC had the highest content of total flavonoid with $1595.86 \mathrm{mg} / 100 \mathrm{~g}$, and the TFCs of the other three were $1553.49 \mathrm{mg} / 100 \mathrm{~g}$ (WY), $1173.07 \mathrm{mg} / 100 \mathrm{~g}(\mathrm{XT})$, and $930.08 \mathrm{mg} / 100 \mathrm{~g}(\mathrm{JN})$. The TFCs of lotus plumule from different districts had obvious differences. Especially, the TFC of lotus plumule from GC was almost double that of lotus plumule from JN. In the previous study, Feng et al. [14] determined flavonoids in lotus plumules of 38 lotus cultivars. The sum of all the flavonoids detected ranged from $730 \mathrm{mg} / 100 \mathrm{~g}$ to $1793 \mathrm{mg} / 100 \mathrm{~g}$ dry weight. The results of TFCs in our study were consistent with the study of Feng et al. [14]. Li et al. [15] quantified flavonoids in nine tissue of Nelumbo nucifera Gaertn. including lotus plumule. The materials used in the study were fresh, so it was unsuitable to compare with the results of our study.

The overall chromatograms (Figures 3 and 4) of lotus plumule from four different districts indicated that similar compositions of lotus plumule were detected among the four cultivars. As discussed in the section of identification of flavonoids in lotus plumules, sixteen flavonoids were detected and identified successfully. Sixteen compounds can be classified into five groups, namely, apigenin, luteolin, quercetin, kaempferol, and diosmetin derivatives. Apigenin derivatives tended to be flavonoid $C$-glycosides and luteolin derivatives could form both flavonoid $\mathrm{C}$-glycosides and flavonoid $\mathrm{O}$-glycosides, while the other three groups tended to be flavonoid $\mathrm{O}$-glycosides. The contents of the thirteen peaks were shown in Figure S1 available online at https://doi.org/10.1155/2017/ 7124354. As it can be seen that peaks 1, 2, 3, 4, 5, 6, and 9, which were all flavonoid $C$-glycosides, accounted for a large proportion of the TFC. It was found that the contents of flavonoid $C$-glycosides of lotus plumule from four different 


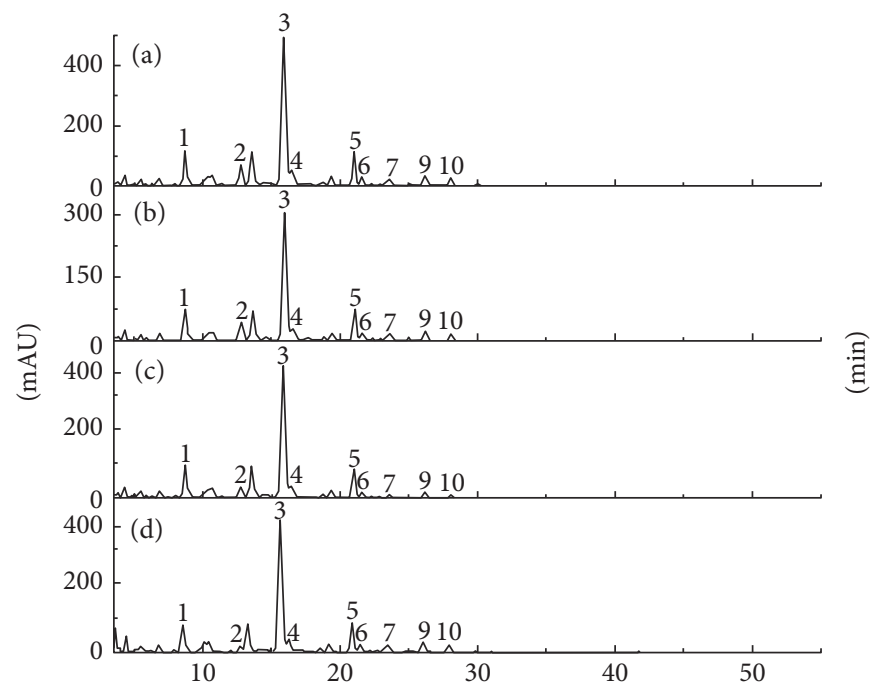

FIGURE 3: Chromatograms of lotus plumule from four different districts: (a) 30\% part for XT; (b) 30\% part for WY; (c) 30\% part for JN; (d) $30 \%$ part for GC.

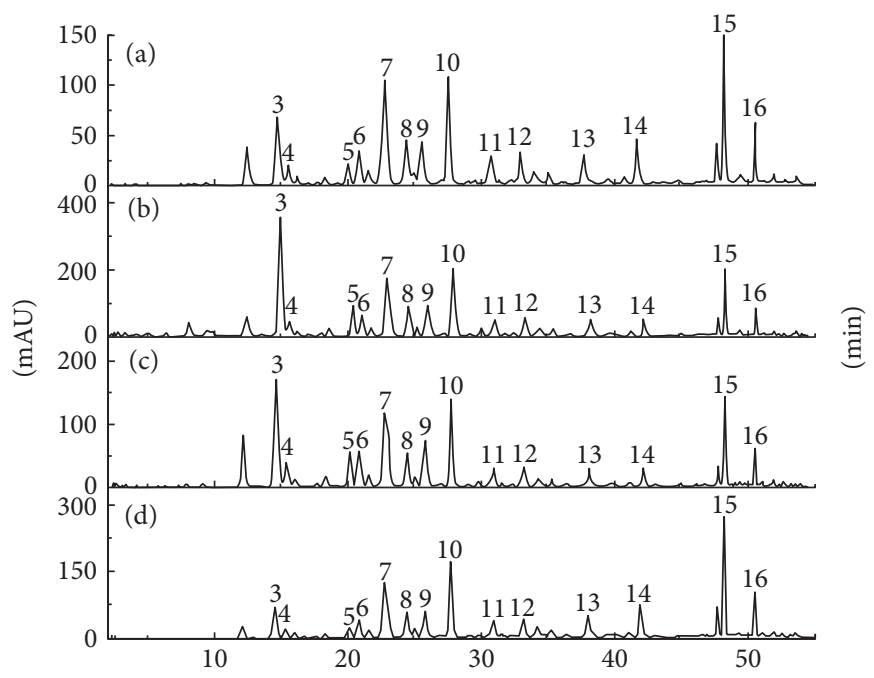

FIGURE 4: Chromatograms of lotus plumule from four different districts: (a) $50 \%$ part for XT; (b) $50 \%$ part for WY; (c) $50 \%$ part for JN; (d) $50 \%$ part for GC.

districts had the similar percentage of the TFC, namely, $80.83 \%$ for GC, $80.91 \%$ for XT, $79.25 \%$ for JN, and $78.53 \%$ for WY. In total, the proportion of flavonoid $C$-glycosides to flavonoid $\mathrm{O}$-glycosides remained unchanged for all these four districts. However, the TFCs and the contents of individual flavonoids between the four districts changed greatly. For example, as can be seen from Table 1 and Figure S1, from peak 1 to peak 13, higher contents of individual flavonoids in lotus plumule from GC were observed than those from the other three districts, which can be used for the differentiation of lotus plumule in GC from the other three districts. In addition, it could be also used for the selection of the best cultivars for future cultivation and applications.

Four representative lotus cultivars were collected from four different places. Both the different environment and cultivars would lead to the changes of flavonoid content. Several reports have documented the influence of environment factors on flavonoid content. Liang et al. [8] studied flavonol compounds from four grape varieties in five regions of China. The results suggested that the relative flavonoid amounts may depend on the cultivar characteristics, but the amounts were more affected by the environmental factors where they were growing. Daniels et al. [11] reported that the content of flavanone in all parts of Gethyllis multifolia decreased when this plant were subjected to photo-stress treatment, while the contents of polyphenols and flavone increased in the roots when subjected to drought stress and the antioxidant activity was also significantly higher. Zhao et al. [24] reported that the total flavonoid content of the safflower leaves increased under $\mathrm{NaCl}$ tress compared to the control group. Wang et al. [25] investigated the influence of temperature and soil moisture on the biosynthesis of 
flavonoids in Ginkgo leaves. It was reported that flavonoid biosynthesis was advantageous in lower temperature and lower soil moisture. In the study of Feng et al. [14], 38 lotus cultivars were from the same places and the results showed great difference of flavonoid content. In fact, plants grown in the natural environment were affected by many integrated factors, which could be used to explain the flavonoid content differences between lotus plumules from four districts. Herein, we conclude that GC is the best place for lotus plumule of high flavonoid content in terms of integrated environment factors. While the similar percentage of flavonoid $C$-glycosides to flavonoid $O$-glycosides among samples from all four districts may be due to their generic backgrounds. Whatsoever, this study revealed for the first time that lotus plumules from different cultivars or from different districts under investigation were only different in the contents of individual flavonoids, but not in the types of individual flavonoids.

\section{Conclusions}

By combining macroporous adsorption resin chromatography with LC-UV/LC-MS, sixteen flavonoids in lotus plumules of four representative lotus cultivars from four different districts were determined and compared. Our results revealed that the studied lotus plumules had similar profiles of flavonoids and similar percentage of flavonoid $C$ glycosides. However, the contents of individual flavonoids differed greatly due to the environment factors, and lotus plumule from GC had the highest content of flavonoid. To some extent, it can be deduced that the environment of GC has the advantage over the other three districts for the synthesis and accumulation of flavonoid in lotus plumule. Furthermore, the method developed in this study would be very useful for both quality assurance and control of lotus plumules, as well as screening new ones with high flavonoid content. These lotus plumules could have high potential in food and pharmaceutical industries since natural flavonoids are deemed beneficial to human health.

\section{Disclosure}

The funders played no roles in the study design, data collection and analysis, and decision to publish the manuscript.

\section{Conflicts of Interest}

The authors declare that there is no conflict of interest regarding the publication of this paper.

\section{Acknowledgments}

This work was jointly supported by the Natural Science Foundation of China (Grant no. 51428303), the "One Hundred Talents Program" from Chinese Academy of Sciences (Grant no. 29Y429291a0129), and the Sino-Africa Joint Research Project (Grant no. SAJC20160233).

\section{References}

[1] C. H. Liao and J. Y. Lin, "Purification, partial characterization and anti-inflammatory characteristics of lotus (Nelumbo nucifera Gaertn) plumule polysaccharides," Food Chemistry, vol. 135, pp. 1818-1827, 2012.

[2] S. Chen, H. H. Zhang, Y. L. Liu, J. B. Fang, and S. H. Li, "Determination of lotus leaf alkaloids by solid phase extraction combined with high performance liquid chromatography with diode array and tandem mass spectrometry detection," Analytical Letters, vol. 46, pp. 2846-2859, 2013.

[3] M. Zhou, M. Jiang, X. Ying et al., "Identification and comparison of anti-inflammatory ingredients from different organs of lotus nelumbo by UPLC/Q-TOF and PCA coupled with a NF$\kappa \mathrm{B}$ reporter gene assay," PLoS One, vol. 8, article e81971, 2013.

[4] J. Shen-Miller, L. H. Aung, J. Turek et al., "Centuries-old viable fruit of sacred lotus Nelumbo nucifera Gaertn var. China antique," Tropical Plant Biology, vol. 6, pp. 53-68, 2013.

[5] Y. Zhang, B. Zheng, Y. Tian, and S. Huang, "Microwaveassisted extraction and anti-oxidation activity of polyphenols from lotus (Nelumbo nucifera Gaertn.) seeds," Food Science and Biotechnology, vol. 21, pp. 1577-1584, 2012.

[6] H. Du, J. Ren, and S. Wang, "Rapid determination of three alkaloids from lotus plumule in human serum using an HPLC-DAD method with a short monolithic column," Food Chemistry, vol. 129, pp. 1320-1324, 2011.

[7] G. P. Xiao, "Development of health beverage of Plumula nelumbinis and Chrysanthemum," Journal of Agriculture, vol. 2, pp. 44-49, 2012.

[8] N. N. Liang, B. Q. Zhu, S. Han et al., "Regional characteristics of anthocyanin and flavonol compounds from grapes of four Vitis vinifera varieties in five wine regions of China," Food Research International, vol. 64, pp. 264-274, 2014.

[9] X. Li, H. Wasila, L. Liu et al., "Physicochemical characteristics, polyphenol compositions and antioxidant potential of pomegranate juices from 10 Chinese cultivars and the environmental factors analysis," Food Chemistry, vol. 175, pp. 575-584, 2015.

[10] M. O. Downey, J. S. Harvey, and S. P. Robinson, "The effect of bunch shading on berry development and flavonoid accumulation in Shiraz grapes," Australian Journal of Grape and Wine Research, vol. 10, pp. 55-73, 2008.

[11] C. W. Daniels, F. Rautenbach, J. L. Marnewick, A. J. Valentine, O. J. Babajide, and W. T. Mabusela, "Environmental stress effect on the phytochemistry and antioxidant activity of a south African bulbous geophyte, Gethyllis multifolia L. Bolus," South African Journal of Botany, vol. 96, pp. 29-36, 2015.

[12] L. S. Yu, Q. Shen, Q. Zhou et al., "In vitro characterization of $\mathrm{ABC}$ transporters involved in the absorption and distribution of liensinine and its analogs," Journal of Ethnopharmacology, vol. 150, pp. 485-491, 2013.

[13] X. X. Zhang, S. C. Ma, P. Mao, and Y. D. Wang, "A new bisbenzylisoquinoline alkaloid from Plumulanelumbinis," Chinese Chemical Letters, vol. 27, pp. 1755-1758, 2016.

[14] C. Y. Feng, S. S. Li, D. D. Yin et al., "Rapid determination of flavonoids in plumules of sacred lotus cultivars and assessment of their antioxidant activities," Industrial Crops and Products, vol. 87, pp. 96-104, 2016.

[15] S. S. Li, J. Wu, L. G. Chen et al., "Biogenesis of C-glycosyl flavones and profiling of flavonoid glycosides in lotus (Nelumbo nucifera)," PLoS One, vol. 9, article e108860, 2014. 
[16] S. Chen, B. H. Wu, J. B. Fang et al., "Analysis of flavonoids from lotus (Nelumbo nucifera) leaves using high performance liquid chromatography/photodiode array detector tandem electrospray ionization mass spectrometry and an extraction method optimized by orthogonal design," Journal of Chromatography. A, vol. 1227, pp. 145-153, 2012.

[17] M. Z. Zhu, T. Liu, C. Y. Zhang, and M. Q. Guo, "Flavonoids of lotus (Nelumbo nucifera) seed embryos and their antioxidant potential," Journal of Food Science, vol. 82, 2017.

[18] M. Z. Zhu, W. Wu, L. L. Jiao, P. F. Yang, and M. Q. Guo, "Analysis of flavonoids in lotus (Nelumbo nucifera) leaves and their antioxidant activity using macroporous resin chromatography coupled with LC-MS/MS and antioxidant biochemical assays," Molecules, vol. 20, pp. 10553-10565, 2015.

[19] Y. Zhang, L. Guo, L. Duan et al., "Simultaneous determination of 16 phenolic constituents in Spatholobi Caulis by high performance liquid chromatography/electrospray ionization triple quadrupole mass spectrometry," Journal of Pharmaceutical and Biomedical Analysis, vol. 102, pp. 110-118, 2015.

[20] Y. Y. Xie, X. Xiao, J. M. Luo et al., "Integrating qualitative and quantitative characterization of traditional Chinese medicine injection by high-performance liquid chromatography with diode array detection and tandem mass spectrometry," Journal of Separation Science, vol. 37, pp. 1438-1447, 2014.

[21] V. Vukics and A. Guttman, "Structural characterization of flavonoid glycosides by multi-stage mass spectrometry," Mass Spectrometry Reviews, vol. 29, pp. 1-16, 2010.

[22] A. Singh, S. Kumar, V. Bajpai, T. J. Reddy, K. B. Rameshkumar, and B. Kumar, "Structural characterization of flavonoid C- and $\mathrm{O}$-glycosides in an extract of Adhatoda vasica leaves by liquid chromatography with quadrupole time-of-flight mass spectrometry," Rapid Communications in Mass Spectrometry, vol. 29, pp. 1095-1106, 2015.

[23] F. Cuyckens and M. Claeys, "Mass spectrometry in the structural analysis of flavonoids," Journal of Mass Spectrometry, vol. 39, pp. 1-15, 2004

[24] G. M. Zhao, Y. Han, X. Sun, S. H. Li, Q. M. Shi, and C. H. Wang, "Salinity stress increases secondary metabolites and enzyme activity in safflower," Industrial Crops and Products, vol. 64, pp. 175-181, 2015.

[25] G. Wang, F. Cao, L. Chang, X. Guo, and J. Wang, “Temperature has more effects than soil moisture on biosynthesis of flavonoids in Ginkgo (Ginkgo biloba L.) leaves," New Forest, vol. 45 , pp. 797-812, 2014. 

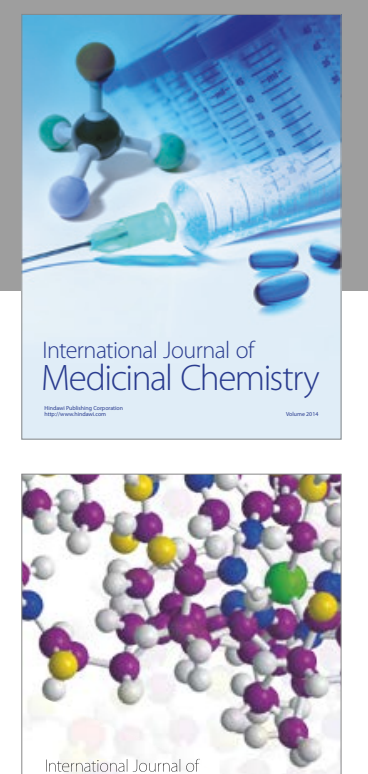

Carbohydrate Chemistry

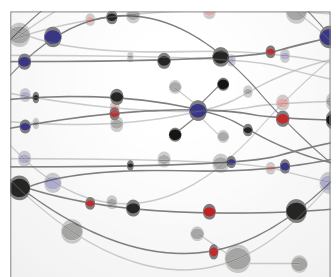

The Scientific World Journal
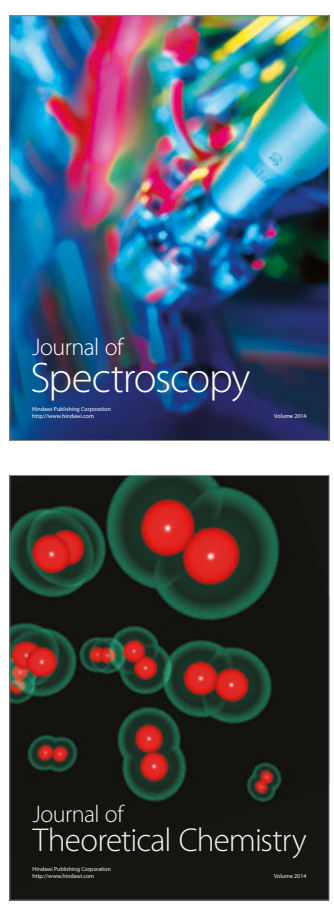
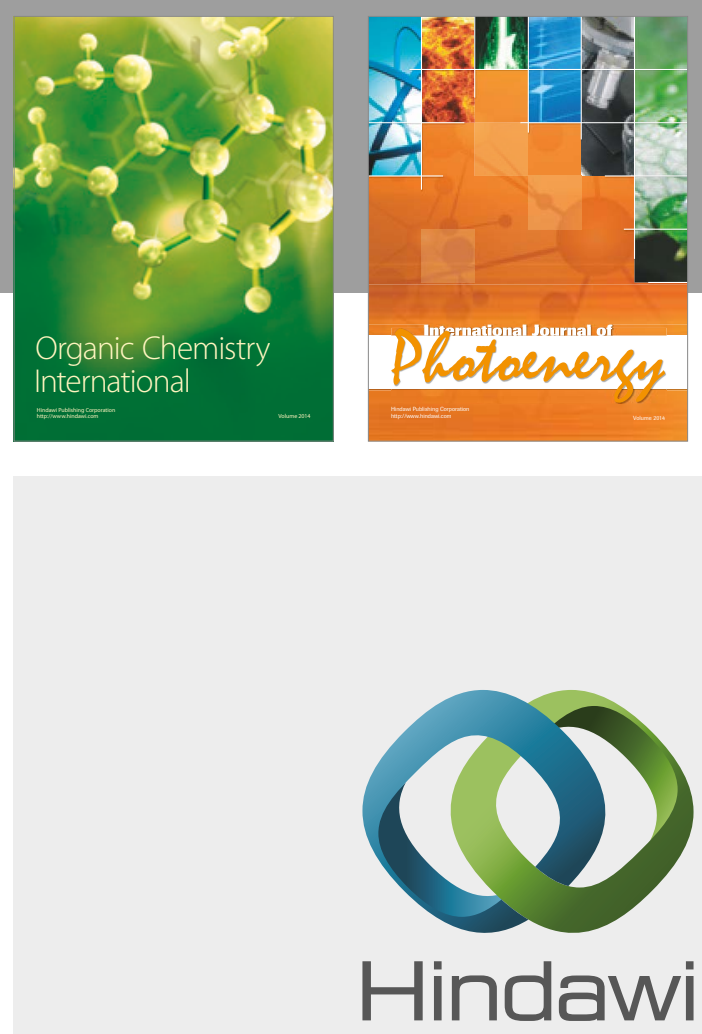

Submit your manuscripts at

https://www.hindawi.com

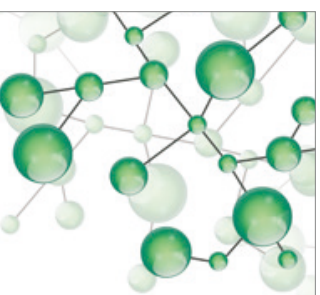

International Journal of

Inorganic Chemistry

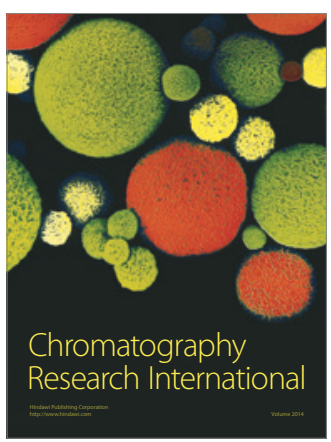

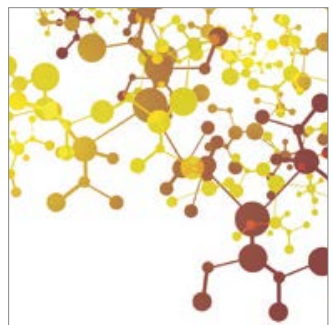

Applied Chemistry
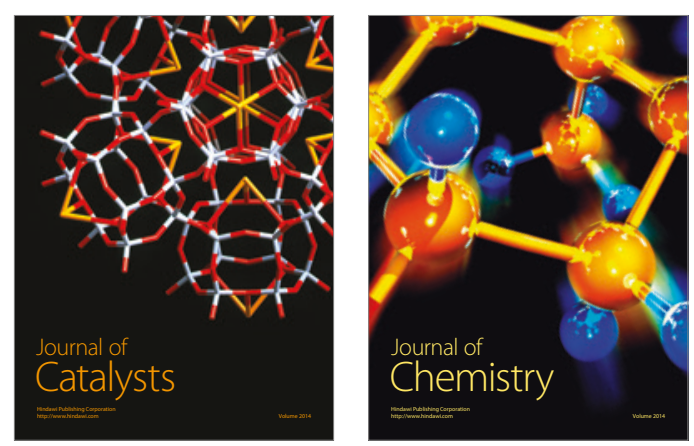
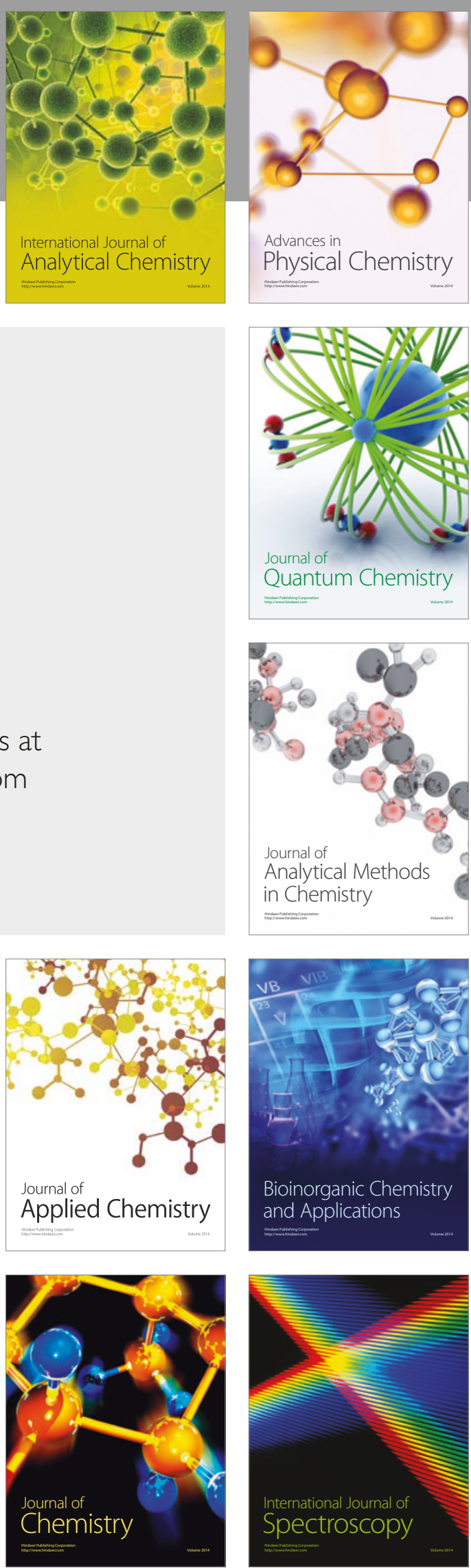